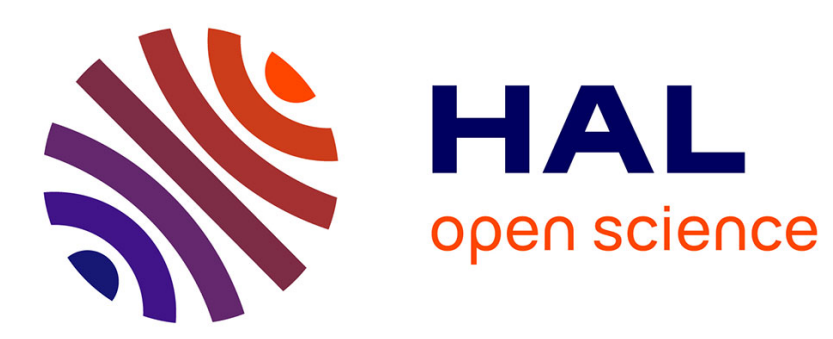

\title{
Porphyre, lecteur et citateur du traité de Plutarque Manger de la viande
}

Fabienne Jourdan

\section{To cite this version:}

Fabienne Jourdan. Porphyre, lecteur et citateur du traité de Plutarque Manger de la viande. Revue des Etudes Grecques, 2005, 118, pp.426-435. 10.3406/reg.2005.4634 . hal-00375411

\section{HAL Id: hal-00375411 \\ https://hal.science/hal-00375411}

Submitted on 2 Jan 2020

HAL is a multi-disciplinary open access archive for the deposit and dissemination of scientific research documents, whether they are published or not. The documents may come from teaching and research institutions in France or abroad, or from public or private research centers.
L'archive ouverte pluridisciplinaire HAL, est destinée au dépôt et à la diffusion de documents scientifiques de niveau recherche, publiés ou non, émanant des établissements d'enseignement et de recherche français ou étrangers, des laboratoires publics ou privés. 


\title{
Porphyre, lecteur et citateur du traité de Plutarque Manger de la
}

\section{viande}

\author{
In: Revue des Études Grecques, tome 118, Juillet-décembre 2005. pp. 426-435.
}

\begin{abstract}
Résumé
Le médio-Platonicien Plutarque n'a pas été lu par les néo- Platoniciens ou du moins est demeuré fort peu apprécié de ces derniers. Seul Porphyre, " parce qu'il n'est pas un homme d'école », le cite abondamment. Son traité sur Y Abstinence, comporte de nombreux parallèles avec le De Esu carnium. Communauté dans le choix des références doctrinales ou de la cible stoïcienne, adoption d'expressions et d'arguments semblables ne se justifient pas seulement par le traitement d'un même sujet, le végétarisme. Porphyre a utilisé le De Esu pour composer le De Abstinentia. Cette certitude nous conduit à penser qu'en III, 18, 3 - 20, 6 , il pourrait avoir cité un passage du traité de Plutarque que la tradition éditoriale de ce dernier n'a pas retenu. L'étude détaillée du texte semblerait confirmer cette hypothèse et inviterait à introduire celui-ci dans une nouvelle édition du De Esu.
\end{abstract}

\section{Abstract}

The middle Platonist Plutarch was not read by the Neo- platonists, or, at any rate, was not much appreciated by them. Porphyry was the only one to quote him abundantly - perhaps because Porphyry himself was not the man of a school. If we compare his treatise De Abstinentia and Plutarchus' treatise on the same topic, De Esu, we find the same doctrinal references, the same target - that is to say the Stoics, and similar expressions or arguments. It is not only due to the fact that they both stood up for vegetarianism. Porphyry had in fact made much use of the De Esu when writing De Abstinentia. This leads us to think that, in De Abstinentia III, 18, 3-20, 6, he might have quoted a passage of Plutarch which does not appear in the current editions of the De Esu. A close examination of this text could confirm this hypothesis and lead us to wish it would appear in a new edition of Plutarch's treatise.

Citer ce document / Cite this document :

Jourdan Fabienne. Porphyre, lecteur et citateur du traité de Plutarque Manger de la viande. In: Revue des Études Grecques, tome 118, Juillet-décembre 2005. pp. 426-435.

doi : 10.3406/reg.2005.4634

http://www.persee.fr/web/revues/home/prescript/article/reg_0035-2039_2005_num_118_2_4634 
Fabienne JOURDAN

\title{
PORPHYRE, LECTEUR ET CITATEUR DU TRAITÉ DE PLUTARQUE MANGER DE LA VIANDE
}

\begin{abstract}
Résumé. - Le médio-Platonicien Plutarque n`a pas été lu par les nćoPlatoniciens ou du moins est demcuré fort peu apprécié de ces derniers. Seul Porphyre. "parce qu'il n'est pas un homme d’école", le cite abondamment. Son traité sur l'Abstinence. comporte de nombreux parallèles avec le De Esı carnilum. Communauté dans le choix des références doctrinales ou de la cible stoïcienne. adoption d'expressions et darguments semblables ne se justifient pas seulement par le traitement d'un même sujet. le végétarisme. Porphyre a utilisé le De Esu pour composer le De Abstinentia. Cette certitude nous conduit à penser qu'en III. 18, $3-20.6$. il pourrait avoir cité un passage du traité de Plutarque que la tradition éditoriale de ce dernier na pas retenu. L'ćtude dćtaillće du texte semblerait confirmer cette hypothèse et inviterait à introduire celui-ci dans une nouvelle édition du De Esu.
\end{abstract}

Abstract. - The middle Platonist Plutarch was not read by the Neoplatonists, or, at any rate, was not much appreciated by them. Porphyry was the only one to quote him abundantly - perhaps because Porphyry himself was not the man of a school. If we compare his treatise De Abstinentia and Plutarchus' treatise on the same topic. De Est, we find the same doctrinal references. the same target - that is to say the Stoics, and similar expressions or arguments. It is not only due to the fact that they both stood up for vegetarianism. Porphyry had in fact made much use of the De Esu when writing De Abstinentia. This leads us to think that. in De Abstinentia III. 18. $3-20,6$, he might have quoted a passage of Plutarch which does not appear in the current editions of the De Esu. A close examination of this text could confirm this hypothesis and lead us to wish it would appear in a new edition of Plutarch`s treatise.

Porphyre est le néo-Platonicien qui a sans doute le plus lu Plutarque, et ce peut-être parce qu'il n'est pas un homme d'école. 
Son traité sur l'Abstinence', dans lequel il utilise nombre d'ouvrages du philosophe chéronéen comme L'Intelligence des animatt.x, L'Utilisation de la raison par les bêtes, Le Banquet des Sept sages, La Vie de Lycurgue, laisse transparaître une bonne connaissance du traité Manger de la viande? ${ }^{2}$. Le projet des deux auteurs n'est pas exactement le même. Porphyre propose une défense argumentée du végétarisme là où Plutarque dresse un vigoureux réquisitoire contre lalimentation carnée. Une comparaison terme à terme des deux ceuvres se révèle donc impossible. Nous pouvons néanmoins relever quelques éléments du De Esı retenus par Purphyre: le renvoi aux mêmes sources doctrinales et surtout le choix d'une même cible privilégiée que sont les Stoïciens: le recours à des expressions. anecdotes et arguments qui semblent directement empruntés à ce traité de Plutarque. Tout cela tend à nous assurer qu'en III, 18, 3-20, 6. Porphyre cite un passage directement tiré de la partie du De Esu qui ne nous est pas parvenue. L'étude attentive du style et de largumentation de ce texte devrait nous en convaincre.

\section{Des références doctrinales semblables et le choix d'une même cible}

Porphyre construit son traité à partir des mêmes sources que Plutarque, quand il ne puise pas directement chez lui. Retenons trois exemples caractéristiques :

a. Pythagore et Empédocle sont évoqués ensemble dans les traités des deux auteurs. Si cela n'a rien d'original dans une exhortation au végétarisme. il s’agit du moins dans les deux cas de fonder le propos sur une tradition religieuse à caractère initiatique.

b. Il semble que Théophraste ait été une source commune. Porphyre compose son deuxième livre à l'aide d'une immense citation du Péripatéticien. Or, en II, 31, 3, la phrase de cet auteur contient plusieurs thèmes scandés dans le De Esı:

«Et quand bien même une telle conduite n'aurait aucun autre résultat. au moins y gagnerions-nous beaucoup quant à l'arrêt mutuel des hosti-

lités. Car ceux dont la sensibilité a refusé de toucher aux autres espèces

1 Toutes les traductions du traité sur L'Abstinence sont empruntées au travail de Michel Patillon. Jean Bouffartigue et Alain-Philippe Segonds dans lédition des Belles Lettres, Paris. 2003. Nous traduisons nous-même en revanche tous les textes de Plutarque.

- Nous désignerons désormais ce traité par son appellation latine abrégée De Esı (carnium).

3 De Estı, 997 c: De Abstincentia. 1. 3. 3: 111. 6. 7. 
de vivants. il est bien évident que l’intellect s’abstiendra de vouloir toucher aux membres de l'espèce (Porphyre. L'Abstinence. II. 31.3 citant Théophraste $)^{+}$. »

Il est permis de penser que Plutarque en a également eu connaissance. Il recourt en effet abondamment à la notion de sensibilité dans son traité. Dès le début (I, 1, 993 a-b), il invite nos cinq sens à éprouver une répugnance commune pour la viande. Il recourt précisément au même verbe ö $\pi \tau \varepsilon \sigma \theta \alpha$, « toucher », qui tend à provoquer une répulsion immédiate. La sensibilité est la première à devoir détourner d'une telle alimentation. Plutarque insiste aussi sur l'idée que nous outrageons de celte manière une autre espèce d'êtres vivants. Il ajoute enfin que la douceur envers les animaux incite nécessairement à la justice entre les hommes:

«Mis à part cela. ne semble-t-il pas qu'il y a là un moyen admirable de fairc naître l'habitude d'une conduite bienveillante à l'égard des hommes ? En effet. qui pourrait commettre une injustice à l'encontre d'un homme. alors qu'envers les êtres étrangers à son espèce. il se comporte avec douceur et humanité ? (De Esı I. 7.995 f-996 a) $)^{5}$."

c. Porphyre recourt à la démonologie dans son deuxième livret. Elle n'est pas exprimée dans les mêmes termes que celle de Plutarque. Nous ne la développerons pas ici, mais noterons seulement qu'elle contribue à le rattacher au courant de pensée médioplatonicien?

Le choix d'une même cible philosophique privilégiée réunit les deux auteurs. Dans son troisième livre. Porphyre répond principalement aux Stoïciens en s’appuyant sur de longues citations de Plutarquc. Il veut clore le débat portant sur nos éventuels devoirs de justice envers les animaux - thèse refutée par les Stö̈ciens. Or, dans son premier livre $(I, 4-6)$, il a déjà emprunté à Plutarque (L'Intelligence des animaux, 964 a c) le développement de l’argumentation anti-végétarienne commune aux Stoïciens et aux Péripatéticiens. Le choix de réfuter en détail les Stoïciens est peut-être inspiré par une lecture du De Esı. A la fin de ce que

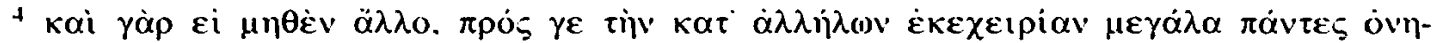

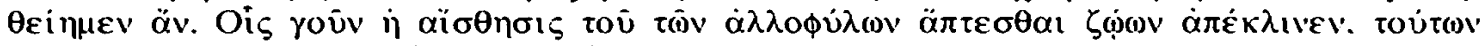

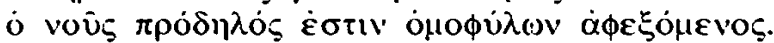

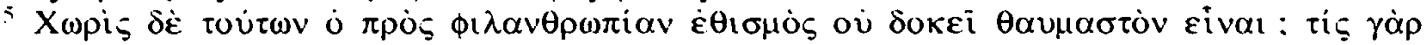

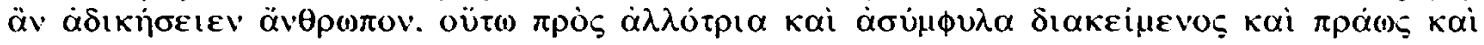
$\phi 1 \lambda \alpha v \theta \rho \omega) \pi(1)$ s.

"De Abstinemia. II. 36. 6-1I. 43.

7 En 11. 40, la mention des épidémics. tremblements de terre et autres catastrophes naturelles ducs aux démons rappelle la responsabilité que leur aurait attribuće Xénocrate daprès Plutarque. De Defectll 14. 417 d.
} 
nous avons reçu comme le « second" » traité (11.7.999 b). Plutarque annonce en effet un examen de la question de la justice envers les animaux. La suite ne nous est malheureusement pas parvenue. Mais il est vraisemblable qu’il entreprend à ce moment la réfutation de la thèse stoïcienne.

\section{Communauté d'expressions, de références cultuelles et histo- riques, d'arguments}

La communauté des sources utilisées par les deux auteurs est corroborée par une tendance à recourir aux mêmes expressions, images et arguments. Dressons-en une liste :

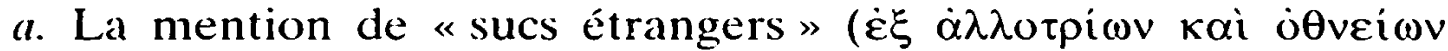
$\chi \cup \mu(\bar{\omega} v$. II. 45, 4) par Porphyre ne peut manquer de faire penser aux "sucs et sérums des blessures mortelles" » du tout début du De Esu (I, 1.993 b), tant l'image est puissante.

b. Lallusion au rite funéraire égyptien qui consiste à retirer les entrailles du mort avant de l'embaumer apparaît dans les deux traités (De Abstinentia IV, 10,3 et 5: De Esu. II, 996 e). Certes. elle n'est pas employée de façon tout à fait identique. Plutarque s'en sert pour illustrer une image. Il dit souhaiter extraire de nous l' «hameçon de l'alimentation carnée ${ }^{10}$ » comme les Égyptiens retiraient l'estomac des cadavres, considéré comme la source de toutes leurs fautes. Cette tradition rituelle est également mentionnée dans le Banquet des Sept Sages, 16. 159 b. Porphyre a peut-être pensé l'expliciter à la lecture du De Esu. Il la fait intervenir dans un développement sur les moyens appliqués par les différentes nations pour préserver la pureté du corps. C'cst dans ce but que les Égyptiens n’embaumaient pas leurs viscères. L’exposé étaye une même défense du végétarisme garant de l'absence de souillure. L'expression de Plutarque pour décrire cette

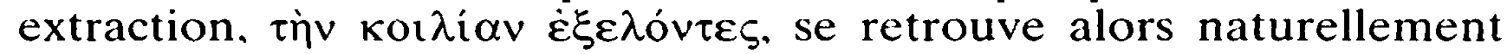
chez Porphyre, ainsi que l'insistance sur la notion de faute par l'emploi du verbe $\dot{\alpha} \mu \alpha \rho \tau \alpha \dot{v} \varepsilon ı v$.

c. La référence à Lycurgue qui se retrouve dans les ouvrages des deux auteurs est particulièrement intéressante. Le passage du quatrième livre de $L^{\prime} A$ bstinence où il est fait référence à la consti-

\footnotetext{
" Le texte a été transmis en deux traités distincts, mais avec U. von Wilamowitz. nous sommes tentée de penser qưil n'y a en réalité qu'un seul traité (voir Ulrich von Wilamowitz-Moellendorf, Kleine Sc/mrifien IV. Academie-Verlag. Berlin. 1962, n" 118. p. 212-217 (originellement paru dans Hermes 40, 1905, p. 161-176).

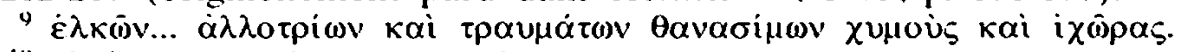

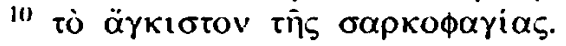


tution lacédémonienne renvoie certes aux Vies (Vie de Lycurgue. 8-10 et 12). Mais là, Plutarque est davantage sensible à l'égalité instaurée par le législateur alors que Porphyre insiste sur le bannis-

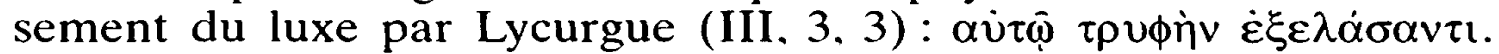
Il ajoute cet élément au passage des Vies dont il se sert. Or, il faut noter que Plutarque évoque déjà l'éviction du luxe par Lycurgue dans le De Esu (II, 2, 997 c-d). Il explique que le législateur aurait interdit les outils trop perfectionnés afin de préserver la sobriété grâce à des constructions rudimentaires. Il se serait agi de bannir tout luxe résultant d'un mauvais genre de vie"l. Porphyre a peut-être pensé attribuer ce bienfait à Lycurgue en lisant le $D e$ Esu avant de développer l'idée à l'aide des Vies.

Certains arguments sont forcément attendus dans un plaidoyer en faveur du végétarisme. Il demeure néanmoins important de relever ceux sur lesquels insistent ensemble les deux auteurs.

a. L'argument de l'habitude intervient plusieurs fois dans le troisième livre du traité sur L'Abstinence. Nous avons déjà noté l'idée chère à Plutarque dans le De Esu selon laquelle la justice envers les animaux conduit à la bienveillance envers les hommes ${ }^{12}$. Elle est renforcée par la mention de l'enseignement que nous auraient transmis Pythagore et Empédocle, nous inculquant l'habitude d'être justes envers les autres espèces ${ }^{13}$. Porphyre cite un passage du traité portant sur L'Intelligence des animaux 2-5 qui exprime exactement la même pensée dans les mêmes termes («Mais les Pythagoriciens faisaient de la bonté envers les bêtes un entraînement à l'humanité et à la pitiélt », De Abstinentia III, 20,7 = De Sollertia 2,959 f). Dans son exhortation finale, il reprend peut-être cet argument développé par Plutarque à la fois dans le De Esu et dans le De Sollertia ( QQui ne voit clairement en effet que le respect de la justice grandit avec la pratique de l'abstinence? Celui qui s'abstient de porter atteinte à tout être animé, même s'il s'agit d'êtres qui n'entrent pas avec lui en société, s'abstiendra à bien plus forte raison de nuire à ses congénères ${ }^{15}{ }^{15}$, De Abstinentia III, 26, 6).

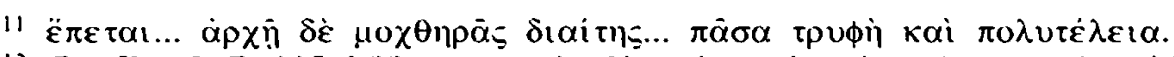

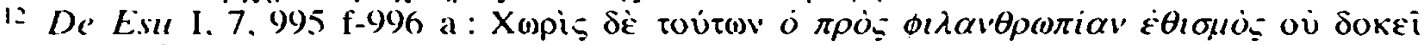

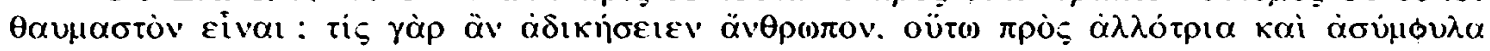

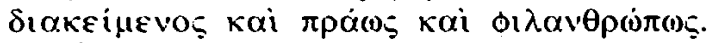

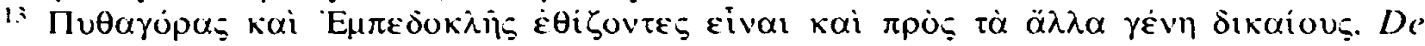
E.su II. 3. 997 c.

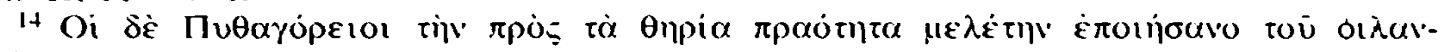

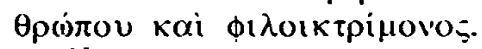

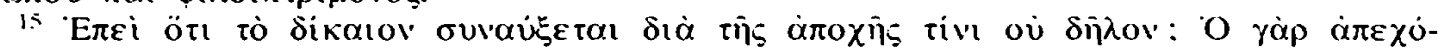

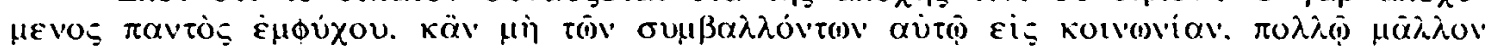

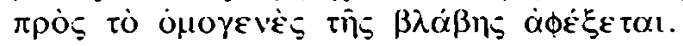


b. Le rappel que les animaux sont des êtres pourvus dune âme (cf. De Esu II, 3) invite peut-être à penser que Porphyre fait une synthèse de cette idée développée par Plutarque dans ses deux traités. Mais nous avons déjà noté que les mêmes arguments ont vraisemblablement été utilisés aussi par Théophrasté.

\section{De l'Abstinence III, 18, 3- 20, 6: un passage du De Esu?}

La communauté de sources, d'expressions et d'idées nous invite à penser que Porphyre a non seulement lu le traité de Plutarque Manger de la viande. mais l'a cité. Dans le troisième livre du $D e$ Abstinentia, il semble en effet reproduire un long passage du Chéronéen qui n'appartient pas à une ceuvre connue de nous ${ }^{16}$ et qui serait vraisemblablement tiré du De Esu. Cette thèse a déjà été soutenue par Bernays qui s`appuyait sur un passage où Plutarque aurait évoqué l'argument stoïcien de l'absence de tout devoir de justice envers les animaux ${ }^{17}$. Ce passage étant malheureusement considéré comme interpolé dans le traité. cetté conjecture a été généralement repoussée (sauf par Wilamowitz ${ }^{18}$ ). Il n èn demeure pas moins que Plutarque a déjà entrepris de réfuter les Stoïciens à la fin de ce qui nous a été transmis comme le "second" traité (II, 6) et que la dernière phrase qui nous est parvenue annonce une discussion sur notre devoir de justice envers les animaux ${ }^{19}$. Lidée que le passage de Plutarque cité par Porphyre comme réponse aux arguments stö̈ciens et péripatéticiens sur l'utilité des animaux et leur exclusion du droit appartienne au $D e$ Esu demeure donc recevable. Une lecture «simultanée » de ce traité et du passage cité suffirait à nous en convaincre. Si les arguments présentés se retrouvent dans dautres ouvrages de Plutarque. il nous semble que le ton du passage est caractéristique du De Esu. La vigueur du réquisitoire contre l'alimentation carnée qui recourt aux images fortes destinées à provoquer le dégoût. aux accumulations d'expressions et d'exemples choquants, aux comparaisons et à l'emploi du raisonnement par l'absurde ou d'une ironie parfois empruntée à Carnéade nous met sur cette piste. Nous retiendrons quelques caractéristiques du texte cité par Porphyre qui rappellent le De Esu.

\footnotetext{
10 Le passage a été recueilli comme fragment 14.5 dans la collection de Bernardakis et fr. 193 dans celle de F. H. Sandbach.

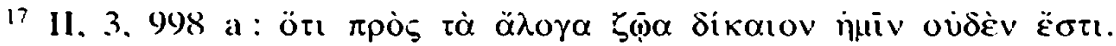

1. "Lesefrüchte" $\mathrm{n}$ " 118, in Hermes 40, 1905, p. 170 $(=$ Kleine Schrifien, t. IV, Berlin, 1962, p. 216).

19 II. 7. 999 b: toūt
} 


\section{a. Remarques de style}

Certaines expressions récurrentes du De Esu apparaissent ici. Elles sont destinées à choquer le lecteur. Relevons par exemple l'emploi du substantif фóvov, «le meurtre, le sang du meurtre »

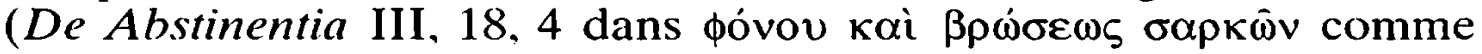

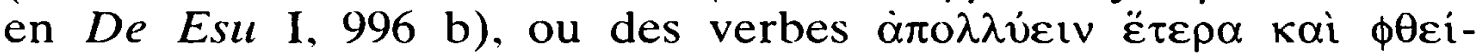

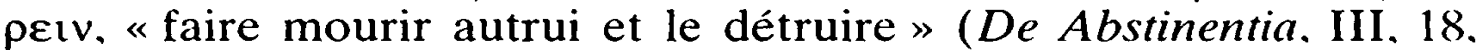
3) pour évoquer la consommation de viande. Le choix des mots est guidé par le projet de condamnation morale comme dans le De Esu. Des alliances de termes propres à choquer sont également présentes. Il est dit par exemple que ce meurtre est commis

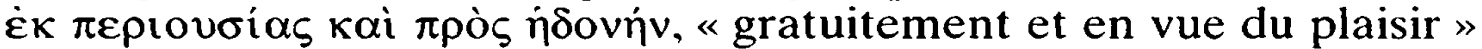
(De Abstinentia 18,3). Un autre substantif caractéristique du De Esu revient constamment dans ce passage. La viande est toujours

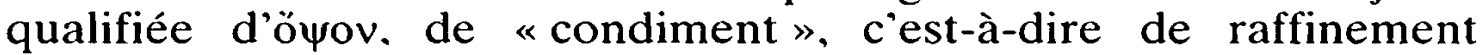
superflu qui s'oppose à un véritable besoin alimentaire. Il apparaît par exemple en III, 19, 1 chez Porphyre dans une nouvelle alliance de termes dénonciatrice puisque le bœuf - animal utile par excellence- devient un simple condiment. L'idée est développée par les termes $\dot{\eta} \delta \dot{\sigma} \sigma \mu \alpha \tau \alpha \pi \lambda \eta \sigma \mu o v \bar{\eta} s$. " condiments de notre goinfrerie 》 et $\kappa \alpha \lambda \lambda \omega \pi i \sigma \mu \alpha \tau \alpha \tau \rho \alpha \pi \dot{\varepsilon} \zeta \eta \zeta$. «ornements de table » que nous pouvons facilement imaginer sous la plume de Plutarque dans le De Esu. Cette phrase tout entière reprend à elle seule plusieurs thèmes de ce traité : la référence à l'abstinence de viande préconisée par Pythagore. l'idée qu'il y a destruction d'une vie. le scandale qui consiste à détruire la vie ${ }^{20}$ pour couvrir nos tables d'« amuse-gueule», si l'on peut dire ${ }^{21}$.

Nous retrouvons aussi dans ce passage le style accumulatif caractéristique du De Esu. Le paragraphe III, 18, 5 du De Abstinentia constitue une immense phrase bâtie sur une comparaison où réapparaît la manière habituelle de Plutarque. Il faut surtout noter l'accumulation de termes accusateurs qui l'inscrirait aisément dans le De Esu: multiplication des motifs honteux ${ }^{22}$. des

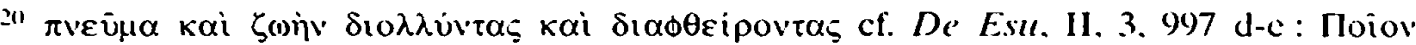

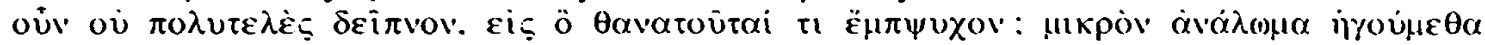
$\Psi \cup \chi \dot{\eta} v$.

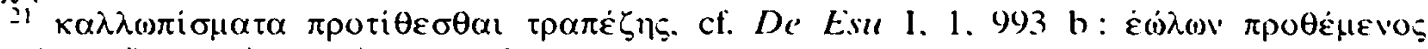

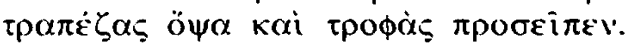

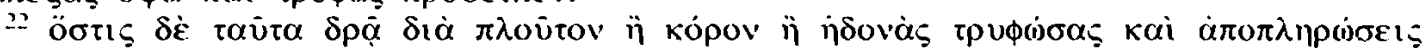

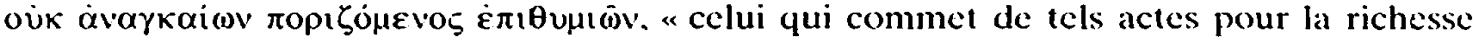
ou la satiété ou pour raffiner ses plaisirs. et se procurer laassouvissement de désirs nullement nécessaires ». 
désignations diffamatoires à l'encontre du consommateur de viande ${ }^{23}$ et finalement, des définitions révélant avec véhémence le caractère répréhensible de son attitude ${ }^{2+}$. Le même genre d'accumulations dénonciatrices se retrouve en III, 19,3 où est dressée une liste de criminels en face d'une séric danimaux innocents, ou en III. 20, 2 où apparaît une suite de coquillages, mollusques et oiseaux prétendument destinés à agrémenter nos plats ( $\zeta \omega \mu$ ò $\kappa \alpha i \quad \pi \alpha \rho \alpha \delta \varepsilon i \pi v i \alpha$, «sauce et desserts»).

Le passage souligne en outre l'absurdité de la thèse adverse. ce que n`a pas manqué de faire Plutarque dans la partie du $D e$ Esu connue de nous. notamment en mettant les Stoïciens en contradiction avec eux-mêmes (II, 6). Ici, le caractère insensé de la position stoïcienne est clairement dénoncé dans la question

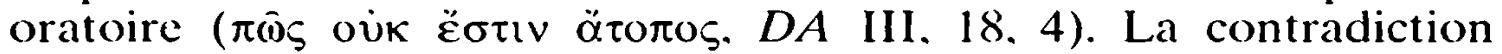
qui consiste à respecter le droit envers des meurtriers et à la refuser à des animaux innoncents est levée dans une gigantesque réduction à labsurde martellée par la construction annulaire de la phrase qui reprend la même question oratoire tout en renchérissant $^{25}$. Largument selon lequel la Providence aurait fait les animaux pour notre usage est renversé par une remarque ironique où se retrouve le vocabulaire du De Esı ("Quant au porc [...]. il n'est né que pour être immolé. Dieu ayant mêlé l'âme à sa chair comme du sel, en vue de nous apprêter un mets friand ${ }^{2 h}$ ", De Abstinentia III, 20, 1). Il est même complètement tourné en dérision par une caractérisation. encore ironique, du dieu qui aurait conçu un tel monde (" [dieu] lemportant ainsi en douceur sur les nourrices et comblant ainsi les régions terrestres de plaisirs et de jouissances ${ }^{27}$ ». De Abstinentia III. 20, 2).

\section{b. Deux arguments clefs du De Esu}

Si le style nous convainc que le passage cité par Porphyre appartient au De Esu, son argumentation vient confirmer cette idée. Dans ce traité en effet, Plutarque insiste sur le rôle de la

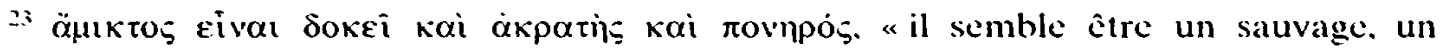
intempérant et un pervers».

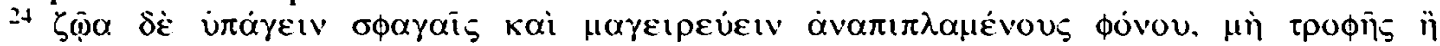

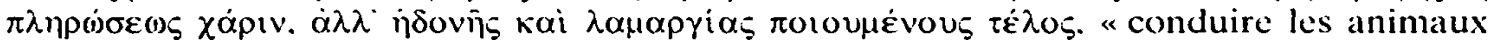
à la boucherie. et. soûl de meurtre. les faire cuire, non pas pour s'en nourrir et s'en rassasicr, mais afin dy trouver son plaisir et den repaître sa gloutonnerie".

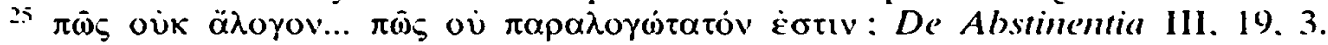

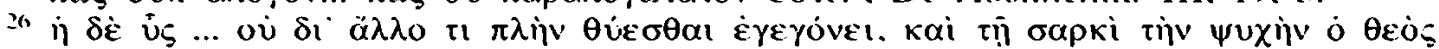

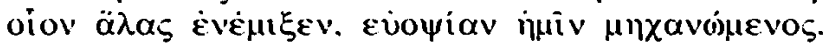

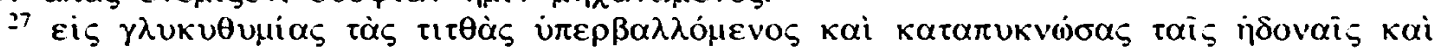

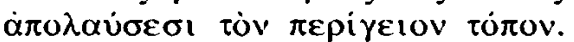


sensation qui doit nous détourner de la viande. Au début du traité. il fait appel aux cinq sens censés éprouver de l'aversion pour une telle alimentation (De Esı I, 1). Ils sont également invités à partager une même répugnance dans le passage cité par Porphyre (De Abstinentia III, 20, 4). Là sont évoqués insectes et reptiles «hideux à voir, sales à toucher, intolérables à sentir. et [qui] poussent des cris effrayants et horribles »: il faut en conclure que de tels animaux ne sont guère appétissants. Les cinq sens sont une fois encore pris à témoin du dégoût que devrait inspirer la viande.

Mais la sensation sert surtout à définir les êtres vivants par opposition aux plantes. Elle permet de distinguer ce qui appartient à l'espèce de ce qui lui est étranger et qu'il faut fuir. Cette distinction stoïcienne apparaît dans le passage de Plutarque cité ici par Porphyre et sert à mettre les Stö̈ciens en contradiction avec eux-mêmes ( et en effet, toute "appropriation" et aversion a son principe dans la sensation. Or, les disciples de Zénon posent l'"appropriation" comme le principe de la justice ${ }^{28} "$, III, 19, 2). Elle est exprimée dans les mêmes termes dans le De Esu II, 3. 997 e ( « Mais quel dîner n'est pas d'un coup excessif quand, pour lui, un être animé doit mourir ? Une âme est-elle à nos yeux une petite dépense? [...] Mais elle a au moins part à la sensation, à la vue, à l'ouïe, à l'imagination, à la compréhension. elle que chaque être reçoit en partage de la nature pour pouvoir obtenir ce qui lui revient en propre et fuir ce qui lui est étranger ${ }^{29} "$ ). Il est permis de supposer que Plutarque réutilise la même idée dans le même traité pour réfuter ses adversaires.

Un autre argument du De Esu récurrent dans ce passage est le caractère excessif de notre cruauté comparée à celle des bêtes sauvages. Dans le texte cité par Porphyre. il est fait mention des individus dénués de raison qui surpassent les animaux féroces par leur violence et démesure ( « [on en voit] un grand nombre encore surpasser par leur cruauté, leur colère et leur avidité les plus redoutables bêtes féroces ${ }^{30}$ ", $D A$ III, 19,3$)$. Ce qui nous a été transmis comme le «premier " traité du De Esı place le même reproche dans la bouche d'un homme appartenant à la seconde

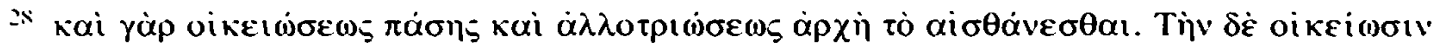

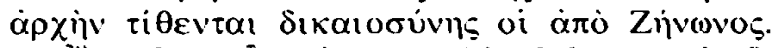

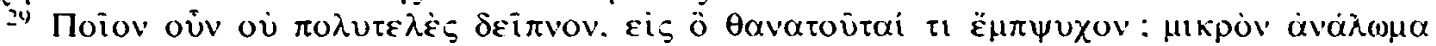

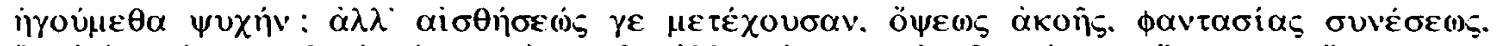

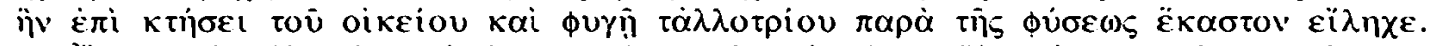

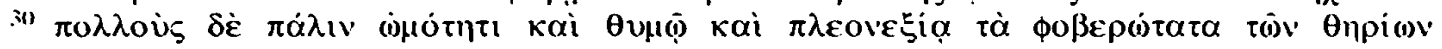

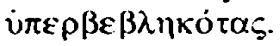


génération - celle qui est soumise à la nécessité - à l'encontre d'un individu de la première qui se serait complu dans la débauche (et la consommation de viande) alors que son époque aurait été celle de l'abondance ("Vous qualifiez les serpent, panthères et lions de sauvages alors que vous vous souillez vous-même par vos meurtres et ne leur laissez rien en cruauté. Car pour eux, le meurtre sert de nourriture, mais pour vous, de condiment ${ }^{31}$ ", $D e$ Esu I. 2. 994 a-b). La fïn du passage cité par Porphyre renchérit exactement sur le même contraste: "[en dévorant les hommes, les animaux féroces ne sont] nullement plus cruels en cela que nous. avec cette différence que c'est le besoin et la faim qui les poussent à cette injustice. tandis que c'est par démesure, par luxe et bien souvent par jeu, au théâtre et dans les chasses, que nous tuons la plupart des animaux (De Abstinentia III, 20,6) , $^{32}$."

La parenté du style et de l'argumentation de ce passage cité par Porphyre avec le De Esu tend à nous convaincre de son appartenance à ce dernier. Nous l'intégrerions volontiers à une réédition du traité.

La reprise des arguments ne constitue pas en soi une preuve de l'appartenance du passage cité par Porphyre dans son traité sur L'Abstinence (III, 18,3-20,6) au De Est. Mais il demeure fort probable que Plutarque a renchéri sur des idées dont il avait déjà esquissé le développement dans la partie du traité qui nous a été transmise. Il les aurait ainsi remises en ceuvre dans la suite du même traité pour réfuter les Stö̈ciens refusant tout devoir de justice envers les animaux. Ce serait à la fois une manière de les marteler, de souligner leur force au cœur même de la polémique tout en conduisant plus aisément le lecteur dans le raisonnement. Ces arguments peuvent évidemment appartenir à d'autres de ses traités. Le seul élément qui nous incite à penser que ce passage puisse réellement être tiré du De Esu demeure la vigueur du style dans le réquisitoire dressé contre l'alimentation carnée.

Fabienne JOURDAN.

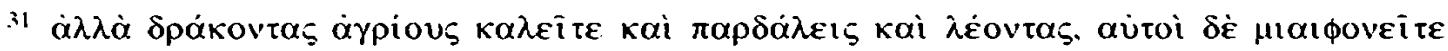

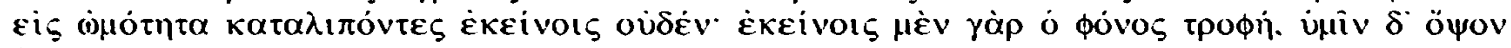
غ̇oriv.

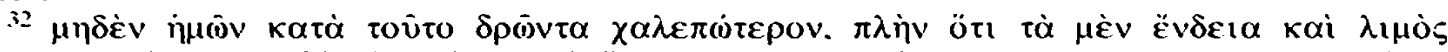

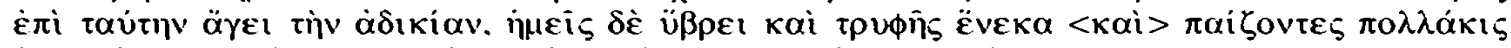

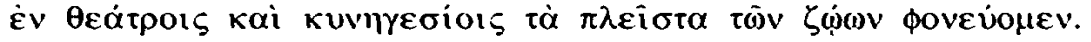

